\title{
Remarkable recent changes in genetic diversity of the avirulence gene AvrStb6 in global populations of the wheat pathogen Zymoseptoria tritici
}

Christopher Stephens ${ }^{1}$, Fatih Ölmez ${ }^{2}$, Hannah Blyth ${ }^{1}$, Megan McDonald ${ }^{3}$, Anuradha Bansal ${ }^{1}$, Emine Burcu Turgay ${ }^{4}$, Florian Hahn ${ }^{1 \#}$, Cyrille Saintenac ${ }^{5}$, Vladimir Nekrasov ${ }^{1}$, Peter Solomon $^{3}$, Andrew Milgate ${ }^{6}$, Bart Fraaije ${ }^{1 \dagger}$, Jason Rudd ${ }^{1}, K$ Kostya Kanyuka ${ }^{1^{*}}$

${ }^{1}$ Rothamsted Research, Harpenden, UK; ${ }^{2}$ Sivas Science and Technology University, Sivas, Turkey; ${ }^{3}$ Australian National University, Canberra, Australia; ${ }^{4}$ Field Crops Central Research Institute, Ankara, Turkey; ${ }^{5}$ Université Clermont Auvergne, INRAE, GDEC, Clermont-Ferrand, France; ${ }^{6} \mathrm{NSW}$ Department of Primary Industries, Wagga Wagga, Australia

\section{Correspondence}

${ }^{*}$ Kostya Kanyuka, Rothamsted Research, Harpenden, UK.

Email: kostya.kanyuka@rothamsted.ac.uk

\section{Present address}

\#Department of Plant Sciences, University of Oxford, Oxford, UK tBart Fraaije, NIAB, Cambridge, UK

\section{SUMMARY}

Septoria tritici blotch (STB), caused by the fungus Zymoseptoria tritici, is one of the most economically important diseases of wheat. Recently, both factors of a gene-for-gene interaction between Z. tritici and wheat, the wheat receptor-like kinase Stb6 and the Z. tritici secreted effector protein AvrStb6, have been identified. Previous analyses revealed a high diversity of AvrStb6 alleles present in historic Z. tritici isolate collections, with up to $\sim 18 \%$ of analysed isolates possessing the avirulence isoform of AvrStb6 identical to that originally identified in the reference isolate IPO323. With Stb6 present in many commercial wheat cultivars globally, we aimed to assess potential changes in AvrStb6 genetic diversity and the incidence of alleles allowing evasion of Stb6-mediated resistance in more recent $Z$. tritici populations. Here we show, using targeted re-sequencing of AvrStb6, that this gene is universally present in field isolates sampled from major wheat-growing regions of the world between 2013-2017. However, in contrast to the data from studies of historic isolates, our study revealed a complete absence of the originally described avirulence isoform of AvrStb6 amongst modern Z. tritici isolates. Moreover, a remarkably small number of alleles, each encoding AvrStb6 protein isoforms conditioning virulence on Stb6-containing wheat, were found to predominate among modern $Z$. tritici isolates. A single virulence isoform of AvrStb6 was found to be particularly abundant throughout the global population. These findings indicate that, despite the ability of $Z$. tritici to sexually reproduce on resistant hosts, AvrStb6 avirulence alleles tend to be eliminated in subsequent populations. 


\section{INTRODUCTION}

The interactions between plant pathogens and their hosts during infection are highly complex and evolutionarily dynamic. Effectors, molecules including proteins that are produced and secreted by pathogens during infection, constitute a vital part of the repertoire of mechanisms utilised in the successful infection of plant hosts (Lo Presti et al., 2015). Effectors function by altering the metabolism of the host plant to facilitate infection, or by suppressing plant immune responses to infection (Djamei et al., 2011; Marshall et al., 2011; Kleemann et al., 2012). However, plants possess a sophisticated innate immune system whose central players are cell surface and intracellular immune receptors including disease resistance $(R)$ genes, which are capable of detecting pathogen effectors and initiating an immune response (Jones and Dangl, 2006; Cook et al., 2015; Kanyuka and Rudd, 2019).

Recognition of a pathogen effector protein by a plant immune receptor thereby introduces an evolutionary pressure on the pathogen to mutate or lose the recognised effector (otherwise known as an avirulence or Avr factor) entirely from its genome. Avr factors may be lost through frameshift or nonsense mutations (Luderer et al., 2002), transposon insertion (Zhang et al., 2015), or repeat-induced point mutations (RIPs) (Rouxel et al., 2011). Indeed, effectors are often located in genomic regions rich in transposon activity which drives effector diversity (Dong et al., 2015). Suppression of Avr factor expression, through mutations in the promoter, histone modification (Soyer et al., 2014) or post-transcriptional silencing (Qutob et al., 2013) may also restore pathogen virulence. In some cases, point mutations in the Avr gene sequence may allow evasion of recognition whilst maintaining protein function (Blondeau et al., 2015; Plissonneau et al., 2017). This may happen in cases where the Avr factor is important for pathogen fitness. Once mutations in Avr genes have occurred that allow evasion of detection by immune receptors, they often spread rapidly through the pathogen population particularly when the cognate disease resistance gene is widely used (Cowger et al., 2000; Hovmøller and Justesen, 2007).

Zymoseptoria tritici, the causal agent of Septoria tritici blotch (STB), is one of the most economically important fungal pathogens of wheat, with fungicide control costs exceeding $€ 1$ billion per year in Europe alone (Torriani et al., 2015). Z. tritici secretes an array of putative effectors during the infection cycle, among which is the avirulence factor AvrStb6 (Zhong et al., 2017; Kema et al., 2018), recognised in a gene-for-gene manner by the cognate cell surface immune receptor Stb6 (Brading et al., 2002; Saintenac et al., 2018). AvrStb6 has been identified in the genomes of the isolates IPO323 and 1E4 collected from the Netherlands and Switzerland in 1981 and 1999, respectively, and recently cloned (Zhong et al., 2017; Kema et al., 2018). The Stb6 gene has also been recently cloned from wheat landrace Chinese Spring and the UK cultivar (cv.) Cadenza (Saintenac et al., 2018). AvrStb6 and Stb6 encode a small, cysteine-rich secreted protein and a wall-associated receptor-like kinase protein, respectively. A functional haplotype of Stb6 conferring resistance to Z. tritici IPO323 is found in over half of commercial cultivars in Europe (Saintenac et al., 2018) and may have been present in wheat populations globally since early in agricultural history (Chartrain et al., 2005). Consequently, pressure on Z. tritici to lose AvrStb6 variants conditioning avirulence on Stb6 containing wheat must be considerable.

There are two previous studies, in which sequencing of AvrStb6 from historic populations of $Z$. tritici has been carried out. In one study, a global population of $Z$. tritici collected between 1990-2001 (Zhan et al., 2005; Brunner and McDonald, 2018) was analysed, whilst the second focussed on a largely French population collected in 2009-2010 (Zhong et al., 
2017). These studies found a high diversity of AvrStb6 alleles, and evidence of positive selection driven by point mutations and recombination. The region of $Z$. tritici chromosome 5 in which AvrStb6 is located was found to be highly dynamic, with extensive transposon activity contributing to AvrStb6 polymorphism (Sánchez-Vallet et al., 2018).

Whilst the AvrStb6 allele distribution in these historic populations have been well characterised, the AvrStb6 allele diversity in more modern Z. tritici populations is unknown. Also unknown is the prevalence of $Z$. tritici isolates which possess AvrStb6 alleles capable of evading Stb6-mediated defence. The specific polymorphisms that drive the change from avirulence to virulence phenotype in the AvrStb6 protein have not yet been determined, although changes at the two amino acid residues (positions 41 and 43) in the AvrStb6 protein have been suggested as being critical for the pathogenicity on wheat cultivars carrying Stb6 (Kema et al., 2018).

In this study, we re-sequenced the AvrStb6 gene from recent field populations of Z. tritici isolates collected between 2013-2017 therefore providing a global snapshot of AvrStb6 allele diversity at this time. We show a notable decrease in the allele diversity of AvrStb6, compared to previous studies, including a recent worldwide shift towards allele encoding Stb6 resistance-breaking isoforms. Interestingly, one particular virulence isoform (I02) was found to be the most abundant in several regions of the world examined. This study therefore provides a rare insight into temporal changes in pathogen effector diversity in response to the host-imposed pressures and highlights the speed with which these changes can occur when a single cognate disease resistance gene is deployed extensively.

\section{RESULTS}

\section{AvrStb6 allele analysis}

Primers flanking the AvrStb6 gene were designed and used for PCR amplification of this gene from a collection of $383 \mathrm{Z}$. tritici isolates sampled from different field grown bread wheat (Triticum aestivum) cultivars in ten countries on five continents. PCR products of expected size were obtained from all analysed samples indicating AvrStb6 is present in all isolates in the collection. Sequencing of the PCR products revealed a total of 52 AvrStb6 alleles (denoted from A01 through to A52; Table S1) coding for 44 protein isoforms (denoted from 101 through to 144; Figure 1). AvrStb6 alleles and the corresponding protein isoforms from the reference isolate IPO323 and another well-studied isolate IPO88004, which are avirulent or virulent on Stb6-containing wheat, respectively, were named as A01/101 and A52/I44.

Almost all alleles were predicted to encode full-length AvrStb6 protein. The exceptions to this were $\mathrm{A} 17$ (only present in four isolates from Turkey) containing a nonsense mutation in the exon 1, and A24 and A46 (each represented by a single isolate also from Turkey) that contained single nucleotide deletions/frameshift mutations located in exon 3 and exon 2 , respectively.

Twenty-nine alleles and twenty-five AvrStb6 protein isoforms each were uniquely present in single isolates in the collection. The majority of recovered AvrStb6 sequences (361 of 381) were 365-bp in length from the ATG start codon to the TGA stop codon and including two introns, with others ranging in lengths from 362-bp to 366-bp. Most of the nucleotide polymorphisms were in exons 2 and 3 , with exon 1 being more conserved, and particularly 
within the sequence coding for the $\mathrm{N}$-terminal signal peptide (Figure 1). Eight alleles (A09, $A 10, A 12, A 21, A 22, A 23, A 37$ and A51) represented by 18 isolates contained three types of in-frame 3-bp deletions in exon 3. These alleles collapse to 7 protein isoforms $(108,109,110$, $111,117,140$ and 143). All these isoforms apart from I17, represented by two isolates from Western Europe, were exclusively found among the $Z$. tritici isolates from Turkey. The identified AvrStb6 isoforms comprised of 80-82 amino acid residues. From these, residues at 52 positions, including all 12 cysteines, were invariant across the collection (excluding the 142 frameshift mutant) (Figure 1). All other amino acid residues in AvrStb6 were variable, with the highest variation identified at positions 41,61 and 77 . As revealed by the amino acid sequence alignment and allele network analysis, AvrStb6 isoforms fell generally into three groups denoted from I, II and III (Figures 1, 2a, and S1). This, in principle, was supported by a phylogenetic analysis, which also identified that group III alleles, largely represented by isolates of Turkish origin, are phylogenetically more distant from those in groups I and II (Figure 2b).

\section{One AvrStb6 isoform predominates among current $Z$. tritici isolates globally}

By far the most prevalent AvrStb6 isoform in the collection was 102, identified in 280 of 381 isolates $(73.5 \%)$. This isoform was the most common almost in every geographic region studied including Australia (96.6\%), Western Europe (91.8\%), USA (82.7\%) and Chile (80\%) (Table 1). The only exceptions were Argentina and Uruguay where isoform 103 was most prevalent, and Turkey where isoform 102 was not identified at all. AvrStb6 isoform 102 was found to be encoded by several alleles: A02 (239 isolates), A03 (34 isolates), A06 (6 isolates), A15 (2 isolates), and A33 (1 isolate). However, in the USA part of the collection, only a single allele (A02) was found to code for $\mathrm{I02.}$

The next most common isoform globally was 103 - the only other isoform to be found in multiple regions - which was identified in 23 isolates (6\% of isolate collection) in Western Europe, South America and Turkey. All other identified AvrStb6 isoforms were represented by between 1 to 8 isolates in the collection, and remarkably well over half of the identified AvrStb6 isoforms $(n=25)$ were each recovered from single isolates (Figure 1).

\section{Turkey is a hotspot of AvrStb6 diversity}

Fifty-seven Z. tritici isolates were sourced from smallholder farmers' wheat fields in Turkey as part of this study. From this sample, 29 AvrStb6 alleles corresponding to 26 protein isoforms were identified. This represents a far higher rate of gene and protein sequence diversity than found in any other region studied here, with more than half of all identified AvrStb6 isoforms being found in Turkey and 25 being unique to this region. The network diagram of AvrStb6 alleles (Figure 2a) visualises this high diversity, with both major branches largely consisting of alleles identified exclusively in the Z. tritici population from Turkey. Interestingly, isoform 102 - the most prevalent isoform globally - was not observed in the Turkish population. This population therefore represents a notable break in the trend described above.

\section{Stb6 resistance-breaking isoforms are widespread in the current population}

We next aimed to determine whether the various identified AvrStb6 isoforms conferred avirulence or virulence towards wheat genotypes carrying the cognate resistance gene Stb6. For this, for each of the nine AvrStb6 isoforms that were identified in more than one $Z$. tritici isolate in the collection (Figure S2) we randomly selected one isolate as representative 
for use in plant infection bioassays. One exception was the most common isoform I02, for which two representative isolates 2NIAB and R16.1 (collected in 2015 and 2016, respectively, from different commercial fields in the UK) were selected. The bioassays involved fungal inoculation of two pairs of wheat genotypes at the seedling stage, with the genotypes in each pair being genetically near identical but distinguished by the presence/absence of Stb6. One pair comprised wheat landrace Chinese Spring carrying the resistance-conferring haplotype 1 of Stb6 (Saintenac et al., 2018) and a near-isogenic line that contains the susceptibility haplotype 3 of Stb6 developed from a cross between Chinese Spring and a susceptible cv. Courtot. The second pair comprised wheat cv. Cadenza (Stb6) and a mutant of cv. Cadenza that contains no functional Stb6 due to a large CRISPR/Cas9-induced frameshift deletion in the first exon of this gene. The previously characterised avirulent and virulent $Z$. tritici isolates IPO323 (Brading et al., 2002) and IPO88004 (Saintenac et al., 2018) possessing isoforms I01 and I44, respectively, were used as controls. Of the ten isolates (representing 9 different isoforms) tested, nine induced typical STB disease symptoms and fungal pycnidia on all tested wheat genotypes irrespectively of the presence/absence of Stb6 (Figures 3 and S3). This included the isolate(s) representing 102, the most prevalent AvrStb6 isoform in our collection.

Other than the avirulent reference isolate IPO323 (I01), only one other isolate could potentially be classified as avirulent on wheat possessing Stb6, as it induced development of some chlorosis but no necrosis nor any asexual sporulation structures (pycnidia) (Figure S3). This isolate possessed the AvrStb6 isoform 113, which shares the highest percent amino-acid identity with the known avirulence isoform I01 (Figure S2). Several isolates, possessing AvrStb6 isoforms classified as virulence, induced lower level of disease on the Stb6-containing wheat compared to their near isogenic genotypes that lack Stb6 as reflected by differences in the observed pycnidospore counts (Figure 3 ). However, these differences were significant (at $p<0.01$ ) only for the Z. tritici isolate T1-A2017.24 possessing the AvrStb6 isoform I13 and the control avirulent isolate IPO323.

\section{Avirulence isoforms of AvrStb6 are common on wheat genotypes irrespective of the Stb6 resistance gene status}

We wondered whether overrepresentation of AvrStb6 virulence isoforms in our isolates collection could have been due to most isolates potentially sampled from resistant, Stb6containing wheat cultivars. The cultivar information was available for 254 out of 381 sampled Z. tritici isolates, and it was possible to obtain 20 cultivars - sources of 210 isolates - for further study (Table S1). Ten of these cultivars have been characterized in the previous study (Saintenac et al., 2018) and their Stb6 haplotype status and reaction to the avirulent strain IPO323 of Z. tritici are known (Table S1). We then re-sequenced Stb6 following the procedure described in Saintenac et al. (2018) from the other ten cultivars including Consort, Dickens, Gallant, Genesis INIA 2375, Kaseberg, KWS Cashel, KWS Lumos, Marston, Solace, and Zulu to identify their Stb6 haplotype status (Table S1). It was determined that 9/20 of these cultivars possess disease resistance conferring haplotype 1 of Stb6 and they were sources of $73(34.8 \%)$ Z. tritici isolates in our collection, whereas the other 11/20 analysed cultivars contained non-functional susceptibility haplotypes 3 or 7 of Stb6 and they represented sources of $137(65.2 \%)$ isolates (Table 2). Therefore, it appears that there was no overall bias in the wheat cultivars used in this study towards susceptible or resistant, and that a larger proportion of $Z$. tritici isolates was sampled from cultivars containing no functional Stb6. 


\section{AvrStb6 expression is highly variable between $Z$. tritici isolates}

Our main hypothesis is that the breakdown of Stb6-mediated resistance by the current $Z$. tritici isolates analysed in this study (Figures 3 and S3) was due to the numerous changes in the AvrStb6 protein sequence identified (Figure 1). However, we also considered an alternative possibility whereby breakdown of Stb6 resistance may have been due to the suppression of AvrStb6 expression during infection. We therefore used a RT-qPCR to compare the expression levels of AvrStb6 in the ten selected Z. tritici isolates (representing ten different most frequent AvrStb6 isoforms) that were virulent on Stb6 wheat versus an avirulent isolate IPO323 during infection of a fully susceptible wheat cv. Taichung 29 that does not possess Stb6 (Ghaffary et al., 2012). As each of the 11 tested isolates has different infection dynamics on this wheat cultivar, the leaf tissues for use in RT-qPCR were sampled at different time points post fungal inoculation, depending on the isolate, when the first symptoms of the disease become visible. This infection phase was chosen as many $Z$. tritici effector genes including AvrStb6 have previously been shown to display maximal expression during this transition to necrotrophy phase (Rudd et al., 2015; Zhong et al., 2017). RT-qPCR analysis revealed that AvrStb6 was expressed in each tested isolate (Figure S4). Moreover, although the AvrStb6 expression levels were highly variable between different isolates, in none of the virulent isolates with the possible exception of WAI1822 carrying AvrStb6 isoform 14 they were substantially lower than in an avirulent isolate IPO323 (Figure S4). This argues against the mechanism of Stb6 resistance breakdown being due to suppression of AvrStb6 expression.

\section{DISCUSSION}

Few studies have analysed the temporal changes in Avr factor prevalence driven by selection pressure from host species in pathogen populations. Often, these have a fairly narrow geographical area coverage. For example, studies into the changes in Avr gene prevalence in the fungal pathogen Leptosphaeria maculans of Brassica crops over time have focussed on single countries such as Canada (Fernando et al., 2018) or Australia (Van de Wouw et al., 2018). On the other hand, studies with a global geographical scope often do not consider the impact of sampling time on changes in Avr factor prevalence. Here we analysed the diversity of the avirulence factor AvrStb6 in the recent global Z. tritici populations. Moreover, we took advantage of the two published similar studies which analysed the population diversity of AvrStb6 in the more historic isolate collections sampled in 1990-2001 or 2009-2010 (Zhong et al., 2017; Brunner and McDonald, 2018). Intercomparing our data that was based on the analysis of isolates sampled in 2013-2017 with that from these two previous studies revealed that large shifts in AvrStb6 allele prevalence have taken place in multiple global regions over a relatively short time period. These include the avirulence isoform of AvrStb6 originally described from the reference isolate IPO323 (collected in the Netherlands in 1981) becoming potentially extinct in the recent $Z$. tritici populations, and emergence of multiple virulence isoforms with one becoming predominant globally. We propose that these changes in Z. tritici populations are imposed by the worldwide extensive deployment of commercial wheat cultivars carrying Stb6 in recent years (Chartrain et al., 2005; Saintenac et al., 2018).

The high proportion of AvrStb6 isoforms to alleles illustrates the high frequency of nonsynonymous mutations within the effector gene, which in turn suggests a strong selection 
pressure for the evolution of novel AvrStb6 protein isoforms that cannot be recognised by the cognate wheat immune receptor Stb6. This phenomenon is well documented for other pathogen effectors, for instance with diversifying selection observed for ToxA, Tox1 and Tox3 of Parastagonospora nodorum (Stukenbrock and McDonald, 2007; McDonald et al., 2013), NIP1 of Rhynchosporium commune (Schürch et al., 2004), and AvrP123 and AvrP4 of the flax rust pathogen Melampsora lini (Barrett et al., 2009). In several fungal pathogens, repeat-induced point (RIP) mutations have been shown to be responsible for the evolution of virulence due to the diversification of effector gene sequences including the introduction of multiple premature stop codons (Fudal et al., 2009). Given the transposon rich, highly dynamic region of the genome in which AvrStb6 is located (Sánchez-Vallet et al., 2018) it seemed possible that a similar mechanism has contributed to the diversification of this $Z$. tritici effector. However, an in-silico analysis suggests a low frequency of RIP-associated mutations in the AvrStb6 genomic region (Figure S5). Moreover, we found that the secretion signal peptide region and all the cysteine residues in AvrStb6 were highly conserved (Figure 1 ), whilst premature stop codon mutations within the gene were extremely rare. The signal peptide of effectors is required for their secretion into the host apoplast, whilst the formation of disulphide bonds between cysteines is thought to help to stabilise the protein in the harsh alkaline $\mathrm{pH}$ apoplastic environment. In addition, using RT-qPCR analysis we showed that AvrStb6 was expressed in a set of $Z$. tritici isolates representing the most frequently occurring isoforms of this effector during a phase transition from biotrophy to necrotrophy (Figure S4). Whilst efforts to demonstrate a role for AvrStb6 isoforms in virulence have to date proved unsuccessful (Kema et al., 2018), these findings combined suggest an evolutionary pressure to maintain a functional effector, implying potential contribution of AvrStb6 to fungal virulence or fitness, perhaps under certain as yet unknown environmental conditions.

Our study revealed the dominance of a single AvrStb6 isoform, 102, in the global population of $Z$. tritici. This isoform was found to be predominant in all countries of Western Europe from which the isolates were sourced, as well as Australia, USA and Chile. It is unknown why this isoform specifically became so prevalent in multiple regions around the world recently, given the ability of isolates carrying other isoforms to also evade Stb6-mediated resistance (Figure 3). However, the limited diversity of Stb6 haplotypes $(n=3)$ in the host wheat plants sampled (Table 2) may suggest that AvrStb6 isoform 102 is specifically adapted for successful infection of wheat possessing these Stb6 haplotypes. The virulence isoform 102 may also be the best-adapted for carrying out its as yet unknown effector function. Notably, sequencing of AvrStb6 from Z. tritici isolates sourced from Oregon, USA in 1990 failed to identify a single occurrence of 102 (Table 1). This is in sharp contrast to our study which identified prevalence of this isoform, found in $82.7 \%$ of the current isolates in the Oregon part of our collection. This population shift, combined with the fact that in all cases 102 was coded by a single allele A02, may suggest the introduction and rapid proliferation of this virulence AvrStb6 allele throughout the population of $Z$. tritici in Oregon, as occurred for example with the recent accidental importation of Pyricularia oryzae, a wheat blast pathogen, from Brazil to Bangladesh (Islam et al., 2016).

Our data also revealed a profound increase in the prevalence of AvrStb6 isoform 102 in the global recent (2013-2017) Z. tritici population compared to that in the older collections comprising isolates sampled in 1990-2001 (Brunner and McDonald, 2018) or 2009-2010 (Zhong et al., 2017) (Table 1). One exception to this is Australia, where a prevalence of AvrStb6 I02 in the local Z. tritici population sampled in 2001 was already evident (Zhan et al., 
2005) (Table 1). Interestingly, in addition to the increasing dominance of a single isoform I02, the overall diversity of AvrStb6 isoforms seem to have decreased over time. Thus, for example, previous studies of historic isolates by Brunner and McDonald (2018) and Zhong et al. (2017) identified thirty and eighteen AvrStb6 isoforms in the populations comprising 142 or 103 isolates, respectively. These numbers are substantially higher than the 42 isoforms identified from 381 isolates analysed in the current study. Such a large shift in the AvrStb6 population diversity that occurred over a relatively short period of time and the increased fixation of AvrStb6 102 in the population could have been caused by the decreased genetic diversity of wheat due to breeding (Fu and Somers, 2009) and widespread global deployment of Stb6-containing cultivars by arable farmers (Chartrain et al., 2005; Saintenac et al., 2018). Other studies have shown changes in the Avr factor prevalence, such as an increased incidence of AvrLm4-7 in Leptosphaeria maculans from 47.2\% in 2005-2006 (Dilmaghani et al., 2009) to 89.7\% in 2010-2011 (Liban et al., 2016) in Western Canada, demonstrating the ability of pathogens to adapt rapidly to changing conditions in host populations. However, to our knowledge this is the first time that a shift of such significance has been shown to occur across a global population of a plant pathogen.

The analysis of AvrStb6 alleles did not identify 102 as the dominant isoform in Argentina and Uruguay, and this isoform was completely absent from the Turkish Z. tritici population. It is possible that the local wheat cultivars used for sampling $Z$. tritici isolates for this study in these countries may have unique genetic makeups, although it was not possible to test this hypothesis in this study. The Turkish population is of particular interest, given it has a substantially higher AvrStb6 allele diversity compared to those in other wheat growing regions studied here. Whilst the exact reasons for this difference are unknown, we hypothesise that the widespread use of local genetically diverse landraces, instead of genetically more similar commercial wheat cultivars, in Turkey is a driver for matching diversification of $Z$. tritici genomes. The location of Turkey near or at the hypothesised geographic region of origin of wheat (Shewry, 2009) and Z. tritici (Stukenbrock et al., 2006) may also be a cause for the high diversity of AvrStb6 alleles observed here because origins of species often also correspond to the centres of their genetic diversity.

A recent study (Kema et al., 2018) suggested that the $Z$. tritici pathogenicity towards wheat cultivars carrying the disease resistance gene Stb6 was associated with changes at the amino acid positions 41 and 43 in the AvrStb6 protein. The new data obtained here support these findings and we confirm that isolates possessing isoforms 101 and I13 having $G$ and $D$ at positions 41 and 43, respectively (Figure S2), were unable to cause disease on wheat cultivars possessing Stb6 (Figure 3). However, we also found that the Z. tritici isolates expressing AvrStb6 isoform 103, 117, or 144, having $E$ or $V$ instead of $G$ at position 41 but $D$ at position 43 were fully virulent on Stb6 wheat (Figure S2). This suggests that amino acid changes in AvrStb6 at position 41 alone may be responsible for the Stb6 resistance breaking ability of $Z$. tritici isolates. It would be interesting to test this hypothesis through targeted mutagenesis in follow on studies.

The number of $Z$. tritici isolates analysed in this study and the failure to identify AvrStb6 isoform 101 in the collection, suggest that this isoform has been eliminated from the modern Z. tritici population worldwide. However, the AvrStb6 allele network analysis indicates that the true diversity of AvrStb6 may be higher than identified as in some cases multiple mutations were found to separate the neighbouring alleles in the network (Figure 2a). A significant amount of undiscovered sequence variation in AvrStb6 may still be present in the global Z. tritici population. However, one of the main conclusions from our study is that the 
wheat disease resistance gene Stb6 has currently been almost completely overcome due to predominance of fungal isolates carrying resistance breaking AvrStb6 isoforms in the modern $Z$. tritici populations around the world. This suggests, that despite the ability of $Z$. tritici to sexually reproduce on resistant hosts (Kema et al., 2018), Avr factors tend to be eliminated in subsequent populations.

\section{EXPERIMENTAL PROCEDURES}

\section{Zymoseptoria tritici isolates collection}

The $381 Z$. tritici isolates used in this study were collected from naturally infected wheat fields in the UK $(n=150)$, Ireland $(n=8)$, France $(n=12)$, Germany $(n=12)$, Australia $(n=$ 58 ) and Turkey $(n=57)$, and from single fields in Chile $(n=10)$, Argentina $(n=10)$, Uruguay $(n=10)$ and USA ( $n=52)$. Most of these isolates were collected between 2015-2017, with the exception of isolates collected in Australia (2014) and Turkey (2013-2016), and the control isolates IPO323 (avirulent on Stb6 wheat) and IPO88004 (virulent on Stb6 wheat) collected in 1981 and 1988 in the Netherlands and Ethiopia respectively (Kema and van Silfhout, 1997; Goodwin et al., 2011). Stocks of Z. tritici isolates were stored as blastospore water suspensions in $50 \%(\mathrm{v} / \mathrm{v})$ glycerol at $-80^{\circ} \mathrm{C}$.

\section{Sequence, diversity, and phylogenetic analysis of AvrStb6}

Genomic DNA extraction was carried out from Z. tritici isolates as previously described (Rudd et al., 2010). Primers for PCR amplification and sequencing of the AvrStb6 gene were designed in the upstream and downstream UTRs of the gene using publicly available and own whole-genome sequencing data for European Z. tritici isolates (Chen et al., Rothamsted Research, Harpenden, UK, 2019, personal communication). Primers avrstb6.f1 and avrstb6.r1 were selected for amplification of AvrStb6 from European isolates, and primers avrstb6.f3 and avrstb6.r1 were used to amplify AvrStb6 from North and South American isolates (Table S2). All amplifications were done using Phusion High-Fidelity DNA Polymerase (New England Biolabs UK Ltd., Hitchin, UK). PCR products were purified using a QIAquick PCR Purification Kit (Qiagen UK Ltd., Manchester, UK) and Sanger sequenced in house or at Eurofins Genomics UK Ltd., Wolverhampton, UK. AvrStb6 sequences from the Australian Z. tritici isolates were extracted from the whole-genome sequencing data (NCBI BioProject accession number PRJNA480739; McDonald et al., 2019).

AvrStb6 allele and the corresponding protein isoform sequences were aligned using MAFFT v.7.388 (Multiple Alignment using Fast Fourier Transform; Katoh and Standley, 2013), and the phylogenetic tree was generated from the allele alignment using PhyML v.3.3.20180621 (Phylogenetic inferences using Maximum Likelihood; Guindon and Gascuel, 2003) with 1000 bootsraps. The AvrStb6 sequence from the Z $Z$. tritici isolate IPO323 was used as a reference for MAFFT alignments, whilst a paralogue of AvrStb6 present on chromosome 10 (gene Mycgr3G82331; Brunner and McDonald, 2018) was used to root the phylogenetic tree. A TCS allele network (Clement et al., 2000) was created to visualise the diversity of AvrStb6 alleles identified in the population using PopArt 1. 7 (Leigh and Bryant, 2015). Coding regions were annotated and translated to amino acid sequence using Geneious 10.2.3 (Biomatters Ltd., Auckland, New Zealand). Repeat-induced point mutation (RIP) analysis of the region of Z. tritici chromosome 5 containing AvrStb6 was carried out using RIPper software (van Wyk et al., 2019). 
The AvrStb6 sequence diversity data obtained here was compared to the similar previously published datasets for the historic $Z$. tritici collections. The AvrStb6 allele sequences obtained in Zhong et al. (2017) were kindly provided by Daniel Croll (University of Neuchâtel, Switzerland) and those obtained in Brunner and McDonald (2018) were downloaded from the NCBI PopSet Database (accession number 1337388362).

\section{Generation of wheat genetic material}

A wheat Chinese Spring near-isogenic line that carries the susceptibility haplotype 3 at the Stb6 locus was obtained following five backcrosses starting with $F_{1}$ Chinese Spring $\times$ Courtot. In each generation, plants were assessed for their responses to the avirulent $Z$. tritici isolate IPO323 and genotyped with simple sequence repeat marker GWM369 to maintain the susceptibility haplotype of $S t b 6$ in the progenies. A single $\mathrm{BC}_{5} \mathrm{~F}_{1}$ plant heterozygous at the Stb6 locus was self-pollinated. $\mathrm{BC}_{5} \mathrm{~F}_{2}$ plants either homozygous for the susceptibility haplotype of Stb6 were selfed and constitute the near-isogenic line used in this study.

The Cadenza $\Delta S t b 6$ mutant was produced by targeting the first exon of the Stb6 gene with CRISPR/Cas9 at five sites (Figure S6). The sgRNA constructs, carrying two sgRNAs each (annotated sequences are available on figshare), were assembled using the pENTR4sgRNA4 vector as previously described (Zhou et al., 2014). All three sgRNA plasmids were co-delivered along with pCas9-GFP (Zhang et al., 2019) encoding the wheat codonoptimised Cas9 fused in frame at the C-terminus with Green Fluorescent Protein (GFP), and pRRes1.111 (Alotaibi et al., 2018) which directs expression of the bar selectable marker gene into immature wheat embryos of wheat cv. Cadenza using biolistics essentially as described in Sparks and Doherty (2020). CRISPR/Cas9 mutagenized $T_{0}$ lines were genotyped for indels using the PCR band-shift assay (Nekrasov et al., 2017). Cas9, sgRNA and bar transgenes were segregated out in subsequent generations and the homozygous Cadenza $\Delta$ Stb6 $\mathrm{T}_{2}$ line was used for phenotyping with $Z$. tritici.

\section{Fungal bioassays}

Leaves of three-week-old wheat seedlings were inoculated with Z. tritici blastospore suspensions at $1 \times 10^{7}$ spores $/ \mathrm{mL}$ as previously described (Keon et al., 2007). Wheat landrace Chinese Spring and cv. Cadenza, both carrying Stb6 were used as resistant controls. A near-isogenic line in the Chinese Spring background that carries a susceptibility haplotype at Stb6 locus, and a CRISPR/Cas9-induced Cadenza $\Delta$ Stb6 mutant were used as susceptible controls. A split plot randomised experimental design was used when carrying out the bioassays.

STB disease symptoms on each inoculated leaf were visually assessed at 21 days post inoculation (dpi) as previously described (Lee et al., 2015). To complement the visual disease assessment, we carried out pycnidiospore wash counts as follows. Approximately 6$\mathrm{cm}$-long segments were cut from the inoculated leaves and these were exposed to high relative humidity at $15^{\circ} \mathrm{C}$ for 48 hours in the dark to induce pycnidiation. Pycnidiospores were washed off the infected leaves by addition of $2 \mathrm{~mL}$ of distilled water supplemented with $0.01 \%$ Tween-20 followed by vortexing for 30 seconds. Optical density at $600 \mathrm{~nm}\left(\mathrm{OD}_{600}\right)$ of the harvested pycnidiospore suspensions were measured by spectrophotometer. This was converted to spores $/ \mathrm{mL}$ by comparing against a standard curve produced by measuring $\mathrm{OD}_{600}$ of control suspensions containing defined pycnidiospore numbers as counted by using a haemocytometer. 


\section{Wheat Stb6 haplotype analysis}

Stb6 haplotype data for ten wheat cultivars, sources of the Z. tritici isolates used here, was available from the recently published study (Saintenac et al., 2018). For ten other cultivars, with previously unknown Stb6 status, we extracted genomic DNA from 3-week-old wheat plants and re-sequenced Stb6 exons following previously published methodology (Saintenac et al., 2018). For Z $Z$. tritici isolates from Australia, Turkey and a few from Western Europe, it was not possible to establish from which cultivar they were sampled.

\section{Analysis of AvrStb6 expression during the infection of wheat}

Three-week-old seedlings of wheat cv. Taichung 29, which is highly susceptible to Z. tritici and contains no known genes/QTLs for resistance to STB (Ghaffary et al., 2012), were inoculated with Z. tritici spore suspension at $1 \times 10^{7}$ spores $/ \mathrm{mL}$ as previously described (Keon et al., 2007). At the emergence of visible symptoms on the inoculated leaves between 12-16-dpi (depending on the particular isolate), the leaves were harvested and snap-frozen in liquid nitrogen. Total RNA was extracted using TRIzol (Fisher Scientific - UK Ltd, Loughborough, UK) following the manufacturer's instructions, and any potential traces of genomic DNA were removed using TURBO DNA-free kit (Fisher Scientific - UK Ltd). First strand cDNA was synthesized from $1 \mu \mathrm{g}$ of total RNA using SuperScript IV Reverse Transcriptase (Fisher Scientific - UK Ltd). First strand cDNA preparations were diluted 1:10 with RNase-free water and subjected to RT-qPCR using SYBR Green JumpStart Taq ReadyMix (Merck Life Science UK Ltd., Gillingham, UK), with primers specified in Table S2. Three biological and three technical replicates were carried out for each sample, with relative expression of AvrStb6 to the Z. tritici housekeeping gene glucose-6-phosphate 1dehydrogenase (G6PDH; gene ID Mycgr3G100879) calculated using the $2^{-\triangle \Delta C T}$ method (Livak and Schmittgen, 2001).

\section{ACKNOWLEDGEMENTS}

We are grateful to Suzanne Clarke for her help with the experimental design of plant inoculation bioassays; Maider Abadie and Ana Machado-Wood for their advice and assistance with the AvrStb6 gene expression analysis; and Hongxin Chen for sharing his spectrophotometry-based method of estimating concentration of pycnidiospores in leaf-wash suspensions. Our gratitude goes to many other colleagues for providing materials used in this study, namely Guilherme Rossato-Augusti, and Pilar Diez for the genomic DNA preparations of the UK, European, USA and South American Z. tritici isolates; Daniel Croll for the AvrStb6 alleles data for the historic Z. tritici population analysed in Zhong et al. (2017); Hongxin Chen for the unpublished whole-genome sequences of various European $Z$. tritici isolates; Lucy Hyde and Caroline Sparks for advice and help with wheat transformation; Keith Edwards for sharing the pCas9-GFP construct; Florence Cambon for her help with the development of a nearly-isogenic Chinese Spring wheat line carrying a susceptibility haplotype at the Stb6 locus; Mike Hammond-Kosack for genomic DNA preparations of several wheat cultivars used for Stb6 exons re-sequencing; Martin Quinke and Christina Hagerty for seeds of USA and South American wheat cultivars, respectively; and Javier Palma-Guerrero for the Z. tritici G6PDH-specific RT-qPCR primer sequences. This work was supported by the UK Biotechnology and Biological Sciences Research Council (BBSRC) Nottingham-Rothamsted Doctoral Training Programme grant (BB/M008770/1) and the Institute Strategic Program grant 'Designing Future Wheat' (BB/P016855/1). 


\section{DATA AVAILABILITY STATEMENT}

The sequencing data that support the findings will be openly available in GenBank at https://www.ncbi.nlm.nih.gov/nucleotide/ following an embargo from the date of publication, accession numbers: MT856831-MT856877. Annotated sgRNA construct sequences in the GenBank format are available on figshare at https://doi.org/10.6084/m9.figshare.12964589.v1.

\section{REFERENCES}

Alotaibi, S.S., Sparks, C.A., Parry, M.A.J., Simkin, A.J. and Raines, C.A. (2018) Identification of leaf promoters for use in transgenic wheat. Plants 7, 27.

Barrett, L.G., Thrall, P.H., Dodds, P.N., Merwe, M. van der, Linde, C.C., Lawrence, G.J. and Burdon, J.J. (2009) Diversity and evolution of effector loci in natural populations of the plant pathogen Melampsora lini. Mol. Biol. Evol. 26, 2499-2513.

Blondeau, K., Blaise, F., Graille, M., et al. (2015) Crystal structure of the effector AvrLm4-7 of Leptosphaeria maculans reveals insights into its translocation into plant cells and recognition by resistance proteins. Plant J. 83, 610-624.

Brading, P.A., Verstappen, E.C.P., Kema, G.H.J. and Brown, J.K.M. (2002) A gene-forgene relationship between wheat and Mycosphaerella graminicola, the Septoria tritici blotch pathogen. Phytopathology 92, 439-445.

Brunner, P.C. and McDonald, B.A. (2018) Evolutionary analyses of the avirulence effector AvrStb6 in global populations of Zymoseptoria tritici identify candidate amino acids involved in recognition. Mol. Plant Pathol. 19, 1836-1846.

Chartrain, L., Brading, P.A. and Brown, J.K.M. (2005) Presence of the Stb6 gene for resistance to septoria tritici blotch (Mycosphaerella graminicola) in cultivars used in wheat-breeding programmes worldwide. Plant Pathol. 54, 134-143.

Clement, M., Posada, D. and Crandall, K.A. (2000) TCS: a computer program to estimate gene genealogies. Mol. Ecol. 9, 1657-1659.

Cook, D.E., Mesarich, C.H. and Thomma, B.P.H.J. (2015) Understanding plant immunity as a surveillance system to detect invasion. Annu. Rev. Phytopathol. 53, 541-563.

Cowger, C., Hoffer, M.E. and Mundt, C.C. (2000) Specific adaptation by Mycosphaerella graminicola to a resistant wheat cultivar. Plant Pathol. 49, 445-451.

Dilmaghani, A., Balesdent, M.H., Didier, J.P., et al. (2009) The Leptosphaeria maculans Leptosphaeria biglobosa species complex in the American continent. Plant Pathol. 58, 1044-1058.

Djamei, A., Schipper, K., Rabe, F., et al. (2011) Metabolic priming by a secreted fungal effector. Nature 478, 395-398.

Dong, S., Raffaele, S. and Kamoun, S. (2015) The two-speed genomes of filamentous pathogens: waltz with plants. Curr. Opin. Genet. Dev. 35, 57-65. 
Fernando, W.G.D., Zhang, X., Selin, C., et al. (2018) A six-year investigation of the dynamics of avirulence allele profiles, blackleg incidence, and mating type alleles of Leptosphaeria maculans populations associated with canola crops in Manitoba, Canada. Plant Dis. 102, 790-798.

Fu, Y.-B. and Somers, D.J. (2009) Genome-wide reduction of genetic diversity in wheat breeding. Crop Sci. 49, 161-168.

Fudal, I., Ross, S., Brun, H., Besnard, A.-L., Ermel, M., Kuhn, M.-L., Balesdent, M.-H. and Rouxel, T. (2009) Repeat-induced point mutation (RIP) as an alternative mechanism of evolution toward virulence in Leptosphaeria maculans. Mol. PlantMicrobe Interact. 22, 932-941.

Ghaffary, S.M.T., Faris, J.D., Friesen, T.L., Visser, R.G.F., Lee, T.A.J. van der, Robert, O. and Kema, G.H.J. (2012) New broad-spectrum resistance to Septoria tritici blotch derived from synthetic hexaploid wheat. Theor. Appl. Genet. 124, 125-142.

Goodwin, S.B., M'barek, S. Ben, Dhillon, B., et al. (2011) Finished genome of the fungal wheat pathogen Mycosphaerella graminicola reveals dispensome structure, chromosome plasticity, and stealth pathogenesis. PLoS Genet. 7, e1002070.

Guindon, S. and Gascuel, O. (2003) A simple, fast, and accurate algorithm to estimate large phylogenies by maximum likelihood. Syst. Biol. 52, 696-704.

Hovmøller, M.S. and Justesen, A.F. (2007) Rates of evolution of avirulence phenotypes and DNA markers in a northwest European population of Puccinia striiformis f. sp. tritici. Mol. Ecol. 16, 4637-4647.

Islam, M.T., Croll, D., Gladieux, P., et al. (2016) Emergence of wheat blast in Bangladesh was caused by a South American lineage of Magnaporthe oryzae. BMC Biol. 14, 84.

Jones, J.D.G. and Dangl, J.L. (2006) The plant immune system. Nature 444, 323-329.

Kanyuka, K. and Rudd, J.J. (2019) Cell surface immune receptors: the guardians of the plant's extracellular spaces. Curr. Opin. Plant Biol. 50, 1-8.

Katoh, K. and Standley, D.M. (2013) MAFFT multiple sequence alignment software version 7: improvements in performance and usability. Mol. Biol. Evol. 30, 772-780.

Kema, G.H. and Silfhout, C.H. van (1997) Genetic variation for virulence and resistance in the wheat - Mycosphaerella graminicola pathosystem III. Comparative seedling and adult plant experiments. Phytopathology 87, 266-272.

Kema, G.H.J., Mirzadi Gohari, A., Aouini, L., et al. (2018) Stress and sexual reproduction affect the dynamics of the wheat pathogen effector AvrStb6 and strobilurin resistance. Nat. Genet. 50, 375-380.

Keon, J., Antoniw, J., Carzaniga, R., Deller, S., Ward, J.L., Baker, J.M., Beale, M.H., Hammond-Kosack, K. and Rudd, J.J. (2007) Transcriptional adaptation of Mycosphaerella graminicola to programmed cell death (PCD) of its susceptible wheat host. Mol. Plant-Microbe Interact. 20, 178-193.

Kleemann, J., Rincon-Rivera, L.J., Takahara, H., et al. (2012) Sequential delivery of hostinduced virulence effectors by appressoria and intracellular hyphae of the phytopathogen Colletotrichum higginsianum. PLoS Pathog. 8, e1002643. 
Lee, W.-S., Rudd, J.J. and Kanyuka, K. (2015) Virus induced gene silencing (VIGS) for functional analysis of wheat genes involved in Zymoseptoria tritici susceptibility and resistance. Fungal Genet. Biol. 79, 84-88.

Leigh, J.W. and Bryant, D. (2015) POPART: full-feature software for haplotype network construction. Methods Ecol. Evol. 6, 1110-1116.

Liban, S.H., Cross, D.J., Kutcher, H.R., Peng, G. and Fernando, W.G.D. (2016) Race structure and frequency of avirulence genes in the western Canadian Leptosphaeria maculans pathogen population, the causal agent of blackleg in brassica species. Plant Pathol. 65, 1161-1169.

Livak, K.J. and Schmittgen, T.D. (2001) Analysis of relative gene expression data using real-time quantitative PCR and the $2^{-\triangle \Delta C T}$ method. Methods 25, 402-408.

Luderer, R., Takken, F.L.W., Wit, P.J.G.M. de and Joosten, M.H.A.J. (2002) Cladosporium fulvum overcomes $C f$-2-mediated resistance by producing truncated AVR2 elicitor proteins. Mol. Microbiol. 45, 875-884.

Marshall, R., Kombrink, A., Motteram, J., Loza-Reyes, E., Lucas, J., Hammond-Kosack, K.E., Thomma, B.P.H.J. and Rudd, J.J. (2011) Analysis of two in planta expressed LysM effector homologs from the fungus Mycosphaerella graminicola reveals novel functional properties and varying contributions to virulence on wheat. Plant Physiol. 156, 756-769.

McDonald, M.C., Oliver, R.P., Friesen, T.L., Brunner, P.C. and McDonald, B.A. (2013) Global diversity and distribution of three necrotrophic effectors in Phaeosphaeria nodorum and related species. New Phytol. 199, 241-251.

McDonald, M.C., Renkin, M., Spackman, M., Orchard, B., Croll, D., Solomon, P.S. and Milgate, A. (2019) Rapid parallel evolution of azole fungicide resistance in Australian populations of the wheat pathogen Zymoseptoria tritici. Appl. Environ. Microbiol. 85, e01908-18.

Nekrasov, V., Wang, C., Win, J., Lanz, C., Weigel, D. and Kamoun, S. (2017) Rapid generation of a transgene-free powdery mildew resistant tomato by genome deletion. Sci. Rep. 7, 482.

Plissonneau, C., Blaise, F., Ollivier, B., Leflon, M., Carpezat, J., Rouxel, T. and Balesdent, M.-H. (2017) Unusual evolutionary mechanisms to escape effectortriggered immunity in the fungal phytopathogen Leptosphaeria maculans. Mol. Ecol. 26, 2183-2198.

Presti, L. Lo, Lanver, D., Schweizer, G., Tanaka, S., Liang, L., Tollot, M., Zuccaro, A., Reissmann, S. and Kahmann, R. (2015) Fungal effectors and plant susceptibility. Annu. Rev. Plant Biol. 66, 513-545.

Qutob, D., Patrick Chapman, B. and Gijzen, M. (2013) Transgenerational gene silencing causes gain of virulence in a plant pathogen. Nat. Commun. 4, 1349.

Rouxel, T., Grandaubert, J., Hane, J.K., et al. (2011) Effector diversification within compartments of the Leptosphaeria maculans genome affected by Repeat-Induced Point mutations. Nat. Commun. 2, 202. 
Rudd, J.J., Antoniw, J., Marshall, R., Motteram, J., Fraaije, B. and Hammond-Kosack, K. (2010) Identification and characterisation of Mycosphaerella graminicola secreted or surface-associated proteins with variable intragenic coding repeats. Fungal Genet. Biol. 47, 19-32.

Rudd, J.J., Kanyuka, K., Hassani-Pak, K., et al. (2015) Transcriptome and metabolite profiling of the infection cycle of Zymoseptoria tritici on wheat reveals a biphasic interaction with plant immunity involving differential pathogen chromosomal contributions and a variation on the hemibiotrophic lifestyle definition. Plant Physiol. $167,1158-1185$.

Saintenac, C., Lee, W.-S., Cambon, F., et al. (2018) Wheat receptor-kinase-like protein Stb6 controls gene-for-gene resistance to fungal pathogen Zymoseptoria tritici. Nat. Genet. 50, 368-374.

Sánchez-Vallet, A., Fouché, S., Fudal, I., Hartmann, F.E., Soyer, J.L., Tellier, A. and Croll, D. (2018) The genome biology of effector gene evolution in filamentous plant pathogens. Annu. Rev. Phytopathol. 56, 21-40.

Schürch, S., Linde, C.C., Knogge, W., Jackson, L.F. and McDonald, B.A. (2004) Molecular population genetic analysis differentiates two virulence mechanisms of the fungal avirulence gene NIP1. Mol. Plant-Microbe Interact. 17, 1114-1125.

Shewry, P.R. (2009) Concomitant increases of the developing seed phosphoenolpyruvate carboxylase activity and the seed protein content of field-grown wheat with nitrogen supply. Wheat. J. Exp. Bot. 60, 1537-1553.

Soyer, J.L., Ghalid, M. El, Glaser, N., et al. (2014) Epigenetic control of effector gene expression in the plant pathogenic fungus Leptosphaeria maculans. PLoS Genet. 10, e1004227.

Sparks, C.A. and Doherty, A. (2020) Genetic transformation of common wheat (Triticum aestivum L.) using biolistics. Methods Mol. Biol. 2124, 229-250.

Stukenbrock, E.H., Banke, S., Javan-Nikkhah, M. and McDonald, B.A. (2006) Origin and domestication of the fungal wheat pathogen Mycosphaerella graminicola via sympatric speciation. Mol. Biol. Evol. 24, 398-411.

Stukenbrock, E.H. and McDonald, B.A. (2007) Geographical variation and positive diversifying selection in the host-specific toxin SnToxA. Mol. Plant Pathol. 8, 321-332.

Torriani, S.F.F., Melichar, J.P.E., Mills, C., Pain, N., Sierotzki, H. and Courbot, M. (2015) Zymoseptoria tritici: a major threat to wheat production, integrated approaches to control. Fungal Genet. Biol. 79, 8-12.

van Wyk, S., Harrison, C.H., Wingfield, B.D., De Vos, L., van der Merwe, N.A., and Steenkamp, E.T. (2019) The RIPper, a web-based tool for genome-wide quantification of Repeat-Induced Point (RIP) mutations. PeerJ 7, e7447.

Wouw, A.P. Van de, Howlett, B.J. and Idnurm, A. (2018) Changes in allele frequencies of avirulence genes in the blackleg fungus, Leptosphaeria maculans, over two decades in Australia. Crop Pasture Sci. 69, 20.

Zhan, J., Linde, C.C., Jürgens, T., Merz, U., Steinebrunner, F. and McDonald, B.A. 
(2005) Variation for neutral markers is correlated with variation for quantitative traits in the plant pathogenic fungus Mycosphaerella graminicola. Mol. Ecol. 14, 2683-2693.

Zhang, Z., Hua, L., Gupta, A., Tricoli, D., Edwards, K.J., Yang, B. and Li, W. (2019) Development of an Agrobacterium-delivered CRISPR/Cas9 system for wheat genome editing. Plant Biotechnol. J. 17, 1623-1635.

Zhang, S., Wang, L., Wu, W., He, L., Yang, X. and Pan, Q. (2015) Function and evolution of Magnaporthe oryzae avirulence gene AvrPib responding to the rice blast resistance gene Pib. Sci. Rep. 5, 11642.

Zhong, Z., Marcel, T.C., Hartmann, F.E., et al. (2017) A small secreted protein in Zymoseptoria tritici is responsible for avirulence on wheat cultivars carrying the Stb6 resistance gene. New Phytol. 214, 619-631.

Zhou, H., Liu, B., Weeks, D.P., Spalding, M.H. and Yang, B. (2014) Large chromosomal deletions and heritable small genetic changes induced by CRISPR/Cas9 in rice. Nucleic Acids Res. 42, 10903-10914. 


\section{TABLE AND FIGURE LEGENDS}

Table 1. Prevalence of AvrStb6 isoform 102 in the recent global Zymoseptoria tritici collection $v s$ in the historic collections.

Table 2. Stb6 haplotype data for wheat cultivars used in this study.

Figure 1. Alignment of the AvrStb6 isoform sequences identified in this study. Protein sequences were aligned using MAFFT v7.388. Numbers in brackets represent the number of isolates identified possessing each isoform. Isoforms 101 and 102 have been identified in the historic Zymoseptoria tritici isolates IPO323 and IPO88004 which are avirulent or virulent on Stb6 wheat, respectively. Amino acids synonymous to the 101 sequence are greyed. Missing residues relative to 101 are represented as dashes. The pink arrow annotates the secretion signal peptide, whilst the green bars represent the exons of the coding gene sequence. The three alignment groupings (I, II and III) of the isoforms are indicated by vertical blue lines to the right of the sequences, and invariant cysteine residues are highlighted in yellow.

Figure 2. Analysis of the AvrStb6 alleles distribution in global Zymoseptoria tritici population. (a) Allele network for global AvrStb6 sequences obtained from the TCS analysis. Each node represents a separate allele identified in this study. The node size represents the number of isolates identified possessing the same allele. The nodes are painted in different colour depending on the geographic region(s) from which the isolates of the same node were identified. Connecting lines between nodes denote closely related alleles, with numbers in brackets corresponding to the number of mutations between adjoined alleles. Edges lengths are not proportional to genetic distances. Black unlabelled nodes represent hypothetical common ancestors of related alleles. The three groupings of alleles I, II and III are highlighted by blue ellipses. (b) Phylogenetic tree of the AvrStb6 alleles constructed using PhyML. The tree was rooted using the Z. tritici gene Mycgr3G82331 located on chromosome 10, a paralogue of AvrStb6. Numbers on branches indicate bootstrap scores for each branch, whilst numbers in brackets indicate the number of isolates identified possessing each allele.

Figure 3. Plant inoculation bioassay. Leaves of differential wheat genotypes, wild-type cv. Cadenza containing Stb6 and a CRISPR/Cas9-induced deletion mutant (Cadenza $\Delta$ Stb6) that lacks the functional resistance gene in the same genetic background, were inoculated as young, three-week-old seedlings with a selection of $Z$. tritici isolates possessing different isoforms of AvrStb6. (a) Counts of pycnidiospores washed off the inoculated wheat leaves at 21 days post inoculation (dpi). Asterisks represent isolates with significant $\left({ }^{*}, p<0.05\right)$ or highly significant $\left({ }^{* *}, p<0.005\right)$ differences in pycnidiospore counts between the resistant and susceptible genotypes. The most common isoform 102 was represented by the two isolates (labelled \#1 and \#2) originating from two different geographic regions. (b) and (c) are images of inoculated wheat leaves harvested at $21 \mathrm{dpi}$ and incubated for two days under $\sim 100 \%$ humidity to induce pycnidiation. Scale bar, $10 \mathrm{~mm}$. 


\section{SUPPORTING INFORMATION}

Table S1. Zymoseptoria tritici isolates used in this study.

Table S2. Primers used in this study.

Figure S1. Frequency of each of the identified AvrStb6 alleles along with their geographic origin.

Figure S2. Alignment of AvrStb6 isoforms from Zymoseptoria tritici isolates tested in pathoassays to determine virulence on Stb6 containing wheat.

Figure S3. Plant inoculation bioassay.

Figure S4. Expression levels of different AvrStb6 alleles during Zymoseptoria tritici infection of wheat.

Figure S5. Analysis of repeat induced point mutation frequency at the AvrStb6 locus on Zymoseptoria tritici chromosome 5 using RIPper (http://theripper.hawk.rocks).

Figure S6. Alignment of the part of the Stb6 coding sequence (exon 1) with the corresponding region in the CRISPR/Cas9-induced wheat Cadenza $\Delta$ Stb6 mutant. 


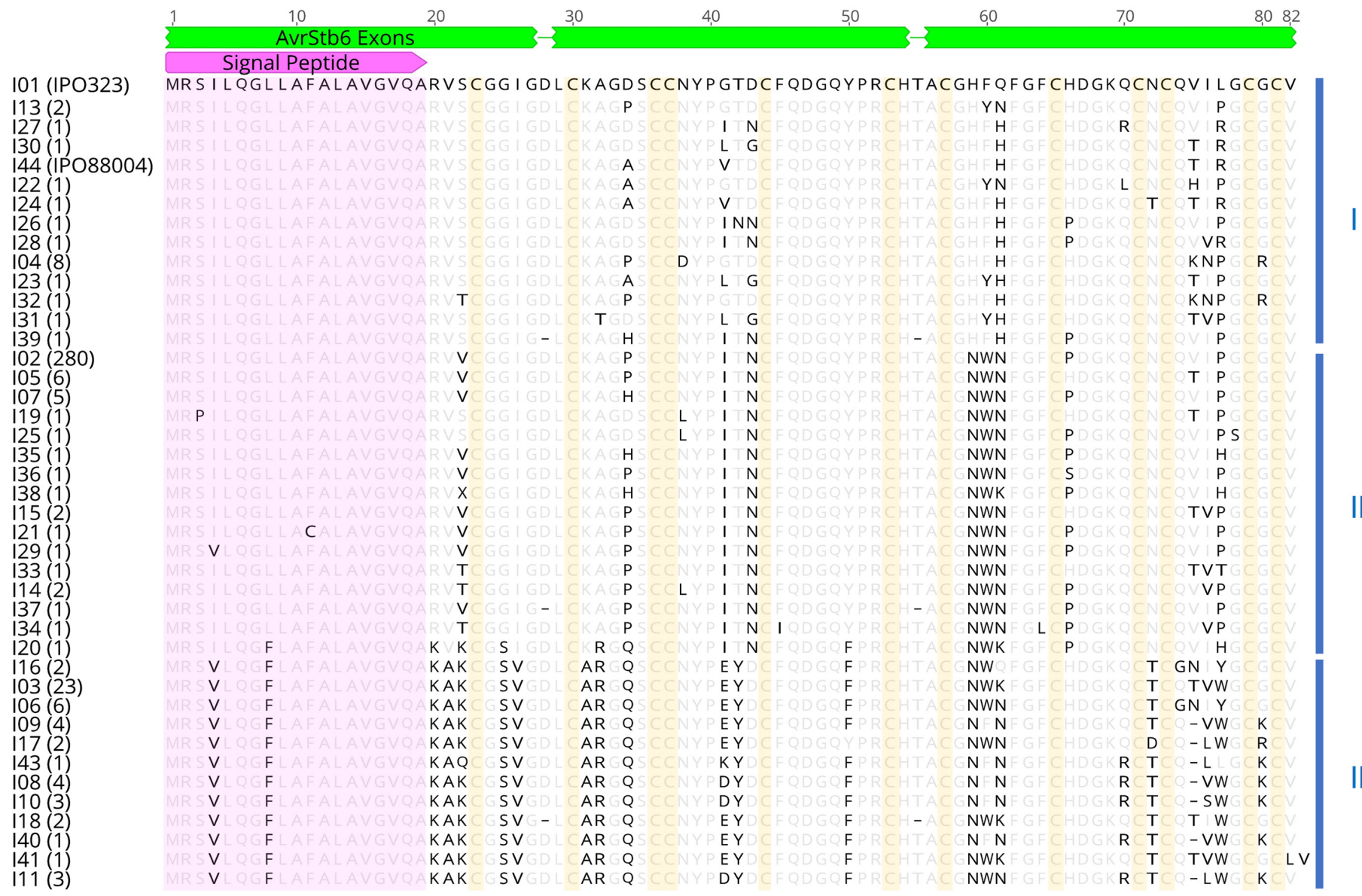

Figure 1. Alignment of the AvrStb6 isoform sequences identified in this study. Protein sequences were aligned using MAFFT v7.388. Numbers in brackets represent the number of isolates identified possessing each isoform. Isoforms 101 and 102 have been identified in the historic Zymoseptoria tritici isolates IPO323 and IPO88004 which are avirulent or virulent on Stb6 wheat, respectively. Amino acids synonymous to the 101 sequence are greyed. Missing residues relative to 101 are represented as dashes. The pink arrow annotates the secretion signal peptide, whilst the green bars represent the exons of the coding gene sequence. The three alignment groupings (I, II and III) of the isoforms are indicated by vertical blue lines to the right of the sequences, and invariant cysteine residues are highlighted in yellow. 


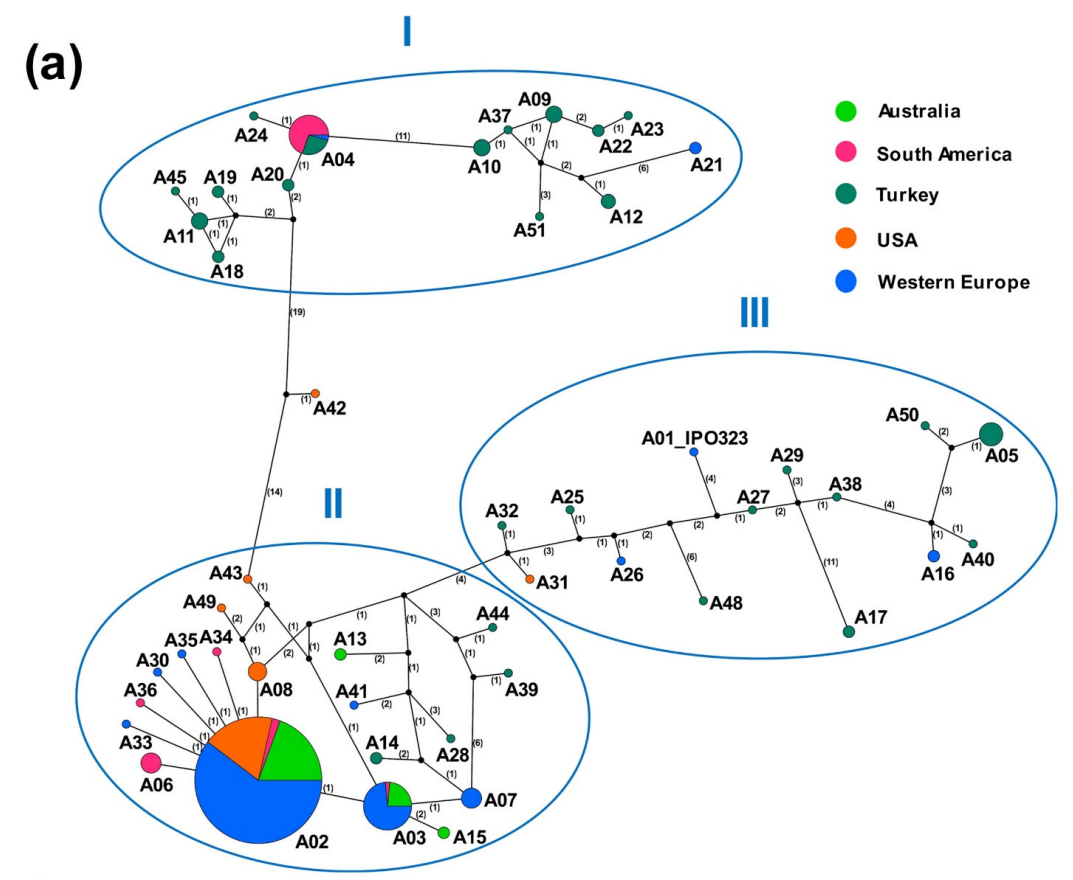

(b)

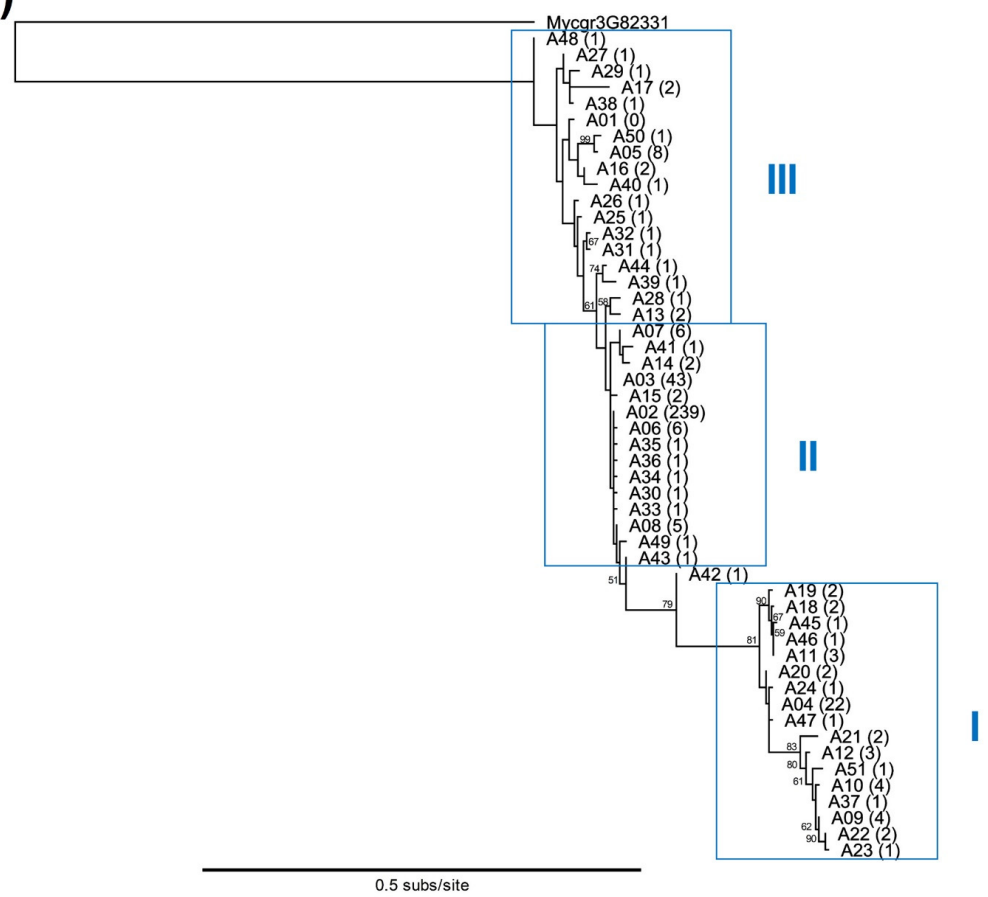

Figure 2. Analysis of the AvrStb6 alleles distribution in global Zymoseptoria tritici population.

(a) Allele network for global AvrStb6 sequences obtained from the TCS analysis. Each node represents a separate allele identified in this study. The node size represents the number of isolates identified possessing the same allele. The nodes are painted in different colour depending on the geographic region(s) from which the isolates of the same node were identified. Connecting lines between nodes denote closely related alleles, with numbers in brackets corresponding to the number of mutations between adjoined alleles. Edges lengths are not proportional to genetic distances. Black unlabelled nodes represent hypothetical common ancestors of related alleles. The three groupings of alleles I, II and III are highlighted by blue ellipses. (b) Phylogenetic tree of the AvrStb6 alleles constructed using PhyML. The tree was rooted using the Z. tritici gene Mycgr3G82331 located on chromosome 10, a paralogue of AvrStb6. Numbers on branches indicate bootstrap scores for each branch, whilst numbers in brackets indicate the number of isolates identified possessing each allele. 


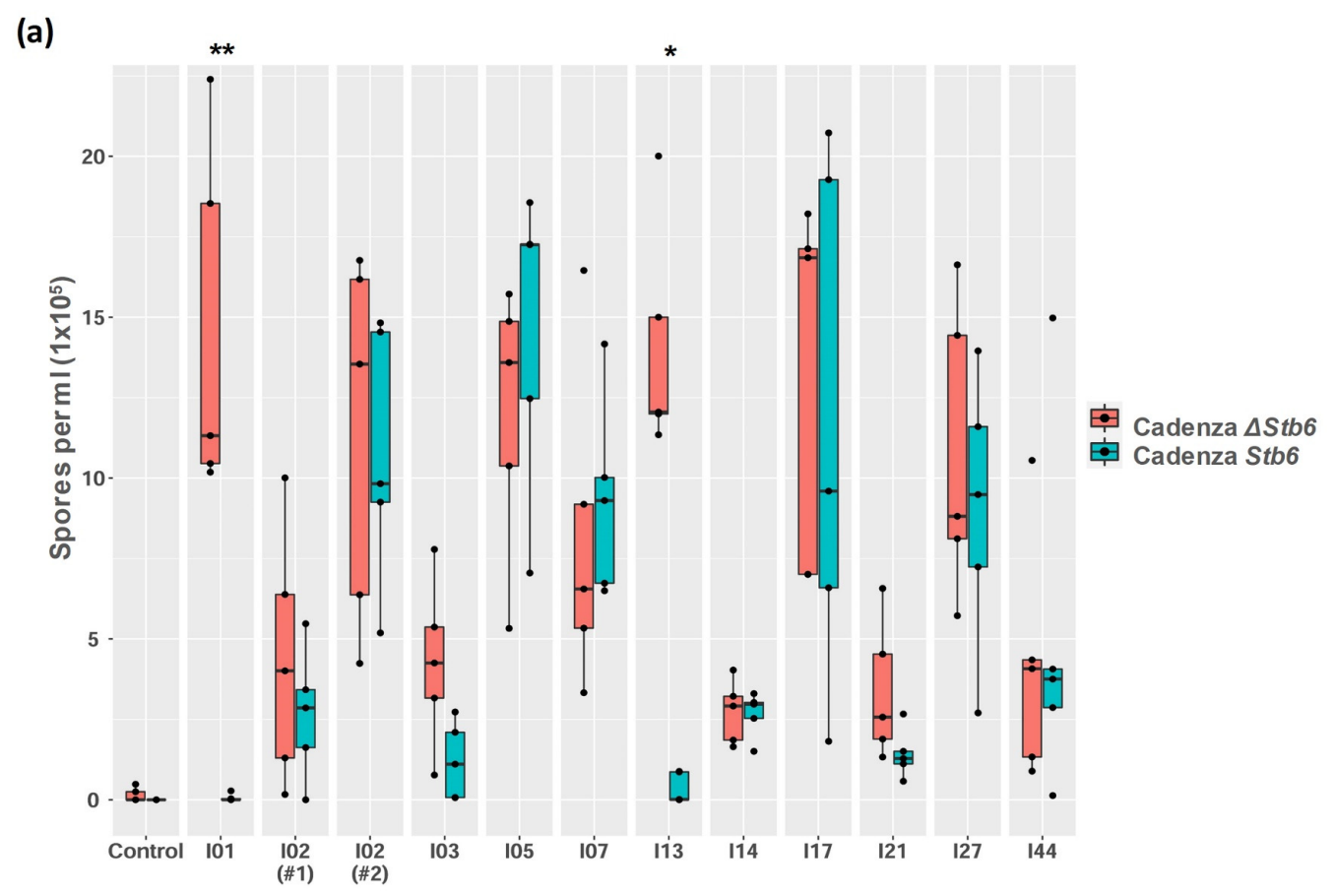

(b)

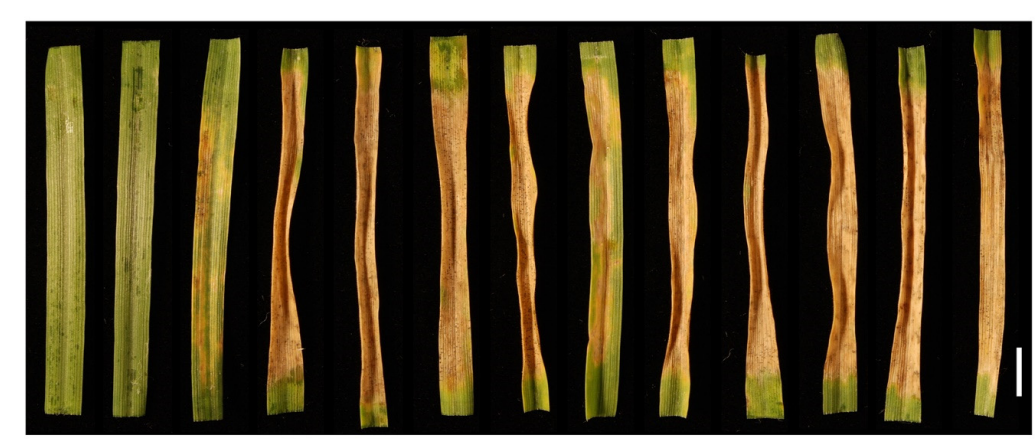

Cadenza

Stb6

(c)
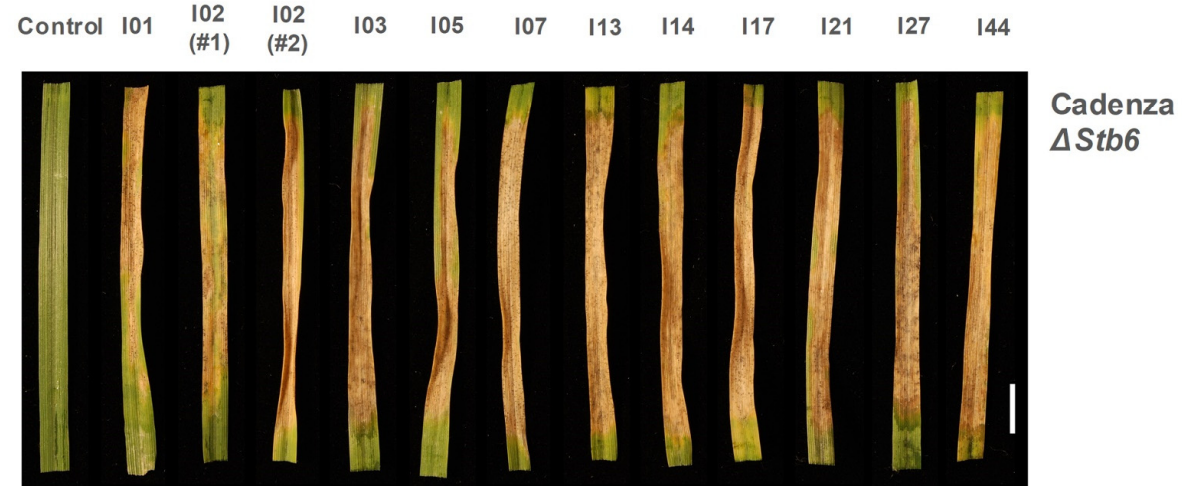

Figure 3. Plant inoculation bioassay. Leaves of differential wheat genotypes, wild-type cv. Cadenza containing Stb6 and a CRISPR/Cas9-induced deletion mutant (Cadenza $\Delta$ Stb6) that lacks the functional resistance gene in the same genetic background, were inoculated as young, three-week-old seedlings with a selection of $Z$. tritici isolates possessing different isoforms of AvrStb6. (a) Counts of pycnidiospores washed off the inoculated wheat leaves at 21 days post inoculation (dpi). Asterisks represent isolates with significant $\left({ }^{*}, p<0.05\right)$ or highly significant $\left({ }^{* *}, p<0.005\right)$ differences in pycnidiospore counts between the resistant and susceptible genotypes. The most common isoform 102 was represented by the two isolates (labelled \#1 and \#2) originating from two different geographic regions. (b) and (c) are images of inoculated wheat leaves harvested at $21 \mathrm{dpi}$ and incubated for two days under $\sim 100 \%$ humidity to induce pycnidiation. Scale bar, $10 \mathrm{~mm}$. 
Table 1. Prevalence of AvrStb6 isoform 102 in the recent global Zymoseptoria tritici collection vs in the historic collections.

\begin{tabular}{|c|c|c|c|c|c|}
\hline Region of Origin & Collection & $\begin{array}{c}\text { Country/State of } \\
\text { origin }\end{array}$ & $\begin{array}{c}\text { Collection } \\
\text { year(s) }\end{array}$ & $\begin{array}{l}\text { Number } \\
\text { of isolates }\end{array}$ & $\begin{array}{c}\text { AvrStb6 } \\
102(\%)\end{array}$ \\
\hline \multirow{7}{*}{ Western Europe } & & England & $2015-2017$ & 135 & 94.8 \\
\hline & & Scotland & 2015-2017 & 14 & 92.9 \\
\hline & this study & Ireland & 2015-2017 & 8 & 87.5 \\
\hline & & France & 2015-2017 & 12 & 91.7 \\
\hline & & Germany & 2015-2017 & 12 & 66.7 \\
\hline & Brunner and McDonald (2018) & Switzerland & 1999 & 29 & 51.7 \\
\hline & Zhong et al. (2017) & France & 2009-2010 & 102 & 49 \\
\hline \multirow{2}{*}{ North America } & this study & USA/Oregon & 2016 & 48 & 89.6 \\
\hline & Brunner and McDonald (2018) & USA/Oregon & 1990 & 56 & 0 \\
\hline \multirow{2}{*}{ Australia } & this study & Tasmania & 2014 & 58 & 96.6 \\
\hline & Brunner and McDonald (2018) & New South Wales & 2001 & 27 & 74.1 \\
\hline \multirow{3}{*}{ South America } & & Chile & 2016 & 10 & 90 \\
\hline & this study & Argentina & 2016 & 10 & 20 \\
\hline & & Uruguay & 2016 & 10 & 30 \\
\hline Mediterranean & this study & Turkey & 2013-2016 & 46 & 0 \\
\hline
\end{tabular}


Table 2. Stb6 haplotype data for wheat cultivars used in this study.

\begin{tabular}{lcc}
\hline Wheat cultivar & $\begin{array}{c}\text { Stb6 } \\
\text { haplotype* }\end{array}$ & $\begin{array}{c}\text { No. of isolates } \\
\text { collected from } \\
\text { the cultivar }\end{array}$ \\
\hline Alchemy & 7 & 1 \\
Consort & 7 & 17 \\
Cordiale & 7 & 13 \\
Cougar & 1 & 22 \\
Crusoe & 7 & 1 \\
Dickens & 1 & 22 \\
Evolution & 1 & 6 \\
Gallant & 7 & 7 \\
Genesis & 3 & 10 \\
JB Diego & 7 & 2 \\
Kaseburg & 7 & 52 \\
KWS Cashel & 3 & 21 \\
KWS Santiago & 1 & 4 \\
KWS Siskin & 1 & 13 \\
Lumos & 7 & 8 \\
Marston & 1 & 1 \\
Reflection & 7 & 5 \\
Solace & 1 & 1 \\
Trapez & 1 & 2 \\
Zulu & 1 & 2 \\
\hline Haplotype & $1^{\#}$ & 73 \\
totals & $3^{\dagger}$ & 31 \\
\hline $7^{\dagger}$ & 106 \\
\hline
\end{tabular}

* Stb6 haplotypes are numbered as in Saintenac et al. (2018)

\# Stb6 haplotype conferring resistance to Z. tritici IPO323

+ Stb6 haplotypes conferring no resistance to Z. tritici IPO323 